\title{
Interacting Holographic Dark Energy in Bianchi Type-V Universe with Variable Deceleration Parameter
}

\author{
Vijay G. Mete1, Vaibhav D. Bokey², Vasant S. Bawane ${ }^{3}$ \\ ${ }^{1}$ R.D.I.K. \& K.D. College, Badnera, India \\ ${ }^{2}$ Nehru Mahavidyalaya, Ner Parsopant, India \\ ${ }^{3}$ M. F. Arts, Comm. \& S. C. Science Mahavidyalaya, Warud, India \\ Email:vmete5622@gmail.com, bokey.vaibhav1@gmail.com, vasantbawane@yahoo.in
}

How to cite this paper: Mete, V.G., Bokey, V.D. and Bawane, V.S. (2019) Interacting Holographic Dark Energy in Bianchi Type-V Universe with Variable Deceleration Parameter. Open Access Library Journal, 6: e5498.

https://doi.org/10.4236/oalib.1105498

Received: May 24, 2019

Accepted: June 11, 2019

Published: June 14, 2019

Copyright $\odot 2019$ by author(s) and Open Access Library Inc.

This work is licensed under the Creative Commons Attribution International License (CC BY 4.0).

http://creativecommons.org/licenses/by/4.0/

\begin{abstract}
The present study deals with spatially homogeneous and anisotropic Bianchi Type- $\mathrm{V}$ universe filled with interacting dark matter and holographic dark energy. The exact solutions of Einstein's equations are obtained by using the variable deceleration parameter in the form $a(t)=(\sinh (\alpha t))^{\frac{1}{n}}$ (Chawla et al. [1]). The physical properties of the model are obtained and discussed in details.
\end{abstract}

\section{Subject Areas \\ Modern Physics, Theoretical Physics}

\section{Keywords}

Bianchi Type-V Universe, Holographic Dark Energy, Interacting Dark Fluids, Variable Deceleration Parameter

\section{Introduction}

The recent remarkable cosmological observations from high red shift Ia supernovae (SNIa) (Perlmutter et al. [2] [3], Riess et al. [4] [5], Astier et al. [6], Spergel et al. [7], Davis et al. [8]) indicate that our universe is accelerating and confirmed later by cross-checks from cosmic microwave background radiation (Bennett et al. [9], Spergel et al. [10]) and large scale structure (Verde et al. [11], Hawkins et al. [12], Abazajian et al. [13] [14] [15], Tegmark et al. [16]) suggests that the universe is spatially flat and dominated by exotic component with large 
negative pressure. This component is usually referred to as dark energy (Weinberg et al. [17], Carroll et al. [18], Peebles et al. [19], Padmanabhan et al. [20]). Astronomical observations indicate that the universe consists of approximately $2 / 3$ dark energy and $1 / 3$ dark matter. The nature of dark energy and dark matter is unknown and many radically different models have been proposed. In order to explain anomalous cosmological observations in cosmic microwave background (CMB) at largest angles, Koivisto et al. [21] have suggested cosmological model with anisotropic and viscous dark energy.

Among all dark energy models, a holographic dark energy (HDE) models have received the remarkable attention (Cohen et al. [22], Horava et al. [23], Thomas et al. [24], Li et al. [25]). According to holographic principle, the number of degrees of freedom in a bounded system should be finite and related to the area of its boundary (Hooft et al. [26]) and with the help of this principle, a field theoretical relation between a short distance (ultraviolet) cut off and a long distance (infrared) cut off was established (Cohen et al. [22]) which ensures that the energy in a box of size $\mathrm{L}$ which has a cosmological length scale, does not exceed the energy of black hole of the same size. Different dark energy models are due to different types of these cut off.

Bianchi models have been studied by several authors to achieve a better understanding of the observed small amount of anisotropy in the universe. The simple Bianchi family containing flat FRW universe as a special case is the type-I space-times. The Bianchi type- $\mathrm{V}$ universe is a generalization of the open universe in FRW cosmology. Hence, its study as dark energy models with non-zero curvature (Coles et al. [27]) in higher dimension is important. Holographic dark energy models have been tested and constrained by various astronomical observations (Zhang et al. [28], Enqvist et al. [29], Shen et al. [30], Chang et al. [31]). The special class models are the models in which holographic dark energy is allowed to interact with dark matter (Carvalho et al. [32], Huang et al. [33], Gong et al. [34] [35], Pavon et al. [36], Wang et al. [37], Perivolaropoulos et al. [38], Nojiri et al. [39], Guberina et al. [40] [41], Guo et al. [42] [43] [44], Hu et al. [45], Li et al. [46], Setare et al. [47] [48], Sadjadi et al. [49], Banerjee et al. [50], Zimdahl et al. [51]). Sarkar et al. [52] [53] [54] have studied non interacting holographic dark energy with linearly varying deceleration parameter in Bianchi type-I and Bianchi type- $\mathrm{V}$ universe and also interacting holographic dark energy in Bianchi type-II.

Spatially homogeneous and anisotropic cosmological models play a significant role in the description of large behavior of the universe, and many authors have been widely studied such models in the search of a relativistic picture of the early universe. Anisotropic Bianchi type-I, Bianchi type-II and Bianchi type-V dark energy models have been extensively studied by (Adhav K. S. [55] [56], Pradhan A. et al. [57]). Kumar and Yadav [58] have constructed some Bianchi type-V cosmological models of accelerating universe with dark energy in general relativity by assuming constant deceleration parameter in order to solve Einstein's field equations. The role of dark energy with variable equation of state parameter 
is studied in details within the evolution of Bianchi type- $\mathrm{V}$ universe and conjointly discovered that dark energy dominates the universe at the present epoch. Pradhan and Amirhashchi [59] have constructed an accelerating dark energy model and explored some new exact solutions of Einstein's field equations in a spatially and anisotropic Bianchi type- $\mathrm{V}$ space-time with minimally interaction of perfect fluid and dark energy components. Adhav et al. [60] explored anisotropic and homogeneous Bianchi type-I universe field with interacting dark matter and holographic dark energy. Som and Sil [61] discussed the general approach of interacting holographic dark energy model.

Motivated by these investigations, we have constructed spatially homogeneous and anisotropic Bianchi type- $\mathrm{V}$ universe field with interacting dark matter and holographic dark energy. In this paper, we obtained the exact solutions of Einstein's field equations by using variable deceleration parameter in the form $a(t)=(\sinh (\alpha t))^{\frac{1}{n}}$.

\section{Metric and Field Equations}

The Bianchi Type- $\mathrm{V}$ metric can be written as

$$
\mathrm{d} s^{2}=\mathrm{d} t^{2}-a_{1}^{2}(t) \mathrm{d} x^{2}-a_{2}^{2}(t) \mathrm{e}^{-2 \beta x} \mathrm{~d} y^{2}-a_{3}^{2}(t) \mathrm{e}^{-2 \beta x} \mathrm{~d} z,
$$

where $a_{1}(t), a_{2}(t)$ and $a_{3}(t)$ are cosmic scale factors and $\beta \neq 0$ is an arbitrary constant.

The Einstein's field equations in natural limit $(8 \pi G=1$ and $c=1)$ are,

$$
R_{i j}-\frac{1}{2} g_{i j} R=-\left({ }^{m} T_{i j}+{ }^{\Lambda} T_{i j}\right),
$$

where

$$
{ }^{m} T_{i j}=\rho_{m} u_{i} u_{j} \text { and }{ }^{\wedge} T_{i j}=\left(\rho_{\Lambda}+p_{\Lambda}\right) u_{i} u_{j}+g_{i j} p_{\Lambda}
$$

are energy momentum tensor for dark matter (pressureless, i.e. $\omega_{m}=0$ ) and holographic dark energy respectively. Here the quantity $\rho_{m}$ is the energy density of dark matter and $\rho_{\Lambda}, p_{\Lambda}$ are energy density and pressure of holographic dark energy respectively.

In co-moving system, the Einstein field Equations (2.2) for the metric (2.1), using Equations (2.3) can be written as

$$
\begin{gathered}
\frac{\dot{a}_{1} \dot{a}_{2}}{a_{1} a_{2}}+\frac{\dot{a}_{2} \dot{a}_{3}}{a_{2} a_{3}}+\frac{\dot{a}_{3} \dot{a}_{1}}{a_{3} a_{1}}-\frac{3 \beta^{2}}{a_{1}^{2}}=\rho_{m}+\rho_{\Lambda}, \\
\frac{\ddot{a}_{2}}{a_{2}}+\frac{\ddot{a}_{3}}{a_{3}}+\frac{\dot{a}_{2} \dot{a}_{3}}{a_{2} a_{3}}-\frac{\beta^{2}}{a_{1}^{2}}=-p_{\Lambda}, \\
\frac{\ddot{a}_{1}}{a_{1}}+\frac{\ddot{a}_{3}}{a_{3}}+\frac{\dot{a}_{1} \dot{a}_{3}}{a_{1} a_{3}}-\frac{\beta^{2}}{a_{1}^{2}}=-p_{\Lambda}, \\
\frac{\ddot{a}_{1}}{a_{1}}+\frac{\ddot{a}_{2}}{a_{2}}+\frac{\dot{a}_{1} \dot{a}_{2}}{a_{1} a_{2}}-\frac{\beta^{2}}{a_{1}^{2}}=-p_{\Lambda},
\end{gathered}
$$




$$
\frac{\dot{a}_{2}}{a_{2}}+\frac{\dot{a}_{3}}{a_{3}}=2 \frac{\dot{a}_{1}}{a_{1}},
$$

where an overhead $\operatorname{dot}\left({ }^{\circ}\right)$ represents derivative with respect to time $t$.

On integrating the Equation (2.8), we obtain

$$
a_{1}^{2}=\lambda a_{2} a_{3}
$$

where $\lambda$ is an integration constant.

On taking $\lambda=1$, without loss of generality, the volume scale factor $V$ and average scale factor $a$ is given by

$$
V=a^{3}=a_{1} a_{2} a_{3}
$$

Subtracting Equation (2.5) from Equation (2.6), Equation (2.6) from Equation (2.7), Equation (2.5) from Equation (2.7) and using Equation (2.10), we get

$$
\begin{aligned}
& \frac{\mathrm{d}}{\mathrm{d} t}\left(\frac{\dot{a}_{1}}{a_{1}}-\frac{\dot{a}_{2}}{a_{2}}\right)+\left(\frac{\dot{a}_{1}}{a_{1}}-\frac{\dot{a}_{2}}{a_{2}}\right) \frac{\dot{V}}{V}=0 \\
& \frac{\mathrm{d}}{\mathrm{d} t}\left(\frac{\dot{a}_{2}}{a_{2}}-\frac{\dot{a}_{3}}{a_{3}}\right)+\left(\frac{\dot{a}_{2}}{a_{2}}-\frac{\dot{a}_{3}}{a_{3}}\right) \frac{\dot{V}}{V}=0 \\
& \frac{\mathrm{d}}{\mathrm{d} t}\left(\frac{\dot{a}_{1}}{a_{1}}-\frac{\dot{a}_{3}}{a_{3}}\right)+\left(\frac{\dot{a}_{1}}{a_{1}}-\frac{\dot{a}_{3}}{a_{3}}\right) \frac{\dot{V}}{V}=0
\end{aligned}
$$

On integrating Equations (2.11a)-(2.11c) and using Equations (2.9) and (2.10), the scale factors $a_{1}(t), a_{2}(t)$ and $a_{3}(t)$ can be written as,

$$
\begin{gathered}
a_{1}(t)=V^{1 / 3} \\
a_{2}(t)=D V^{1 / 3} \exp \left(X \int \frac{\mathrm{d} t}{V}\right), \\
a_{3}(t)=D^{-1} V^{1 / 3} \exp \left(-X \int \frac{\mathrm{d} t}{V}\right)
\end{gathered}
$$

where $X$ and $D$ are constants of integration.

The holographic dark energy density is given by,

$$
\rho_{\Lambda}=3\left(\alpha_{1} H^{2}+\beta_{1} \dot{H}\right)
$$

i.e. $\rho_{\Lambda}=3\left(\alpha_{1} H^{2}+\beta_{1} \dot{H}\right)$ with $M_{p}^{-2}=8 \pi G=1$ (Granda et al. [62]), where $\alpha_{1}$ and $\beta_{1}$ are constants.

For the universe, where dark energy and dark matter are interacting with each other, the total energy density $\rho=\left(\rho_{m}+\rho_{\Lambda}\right)$ satisfies the equation of continuity as,

$$
\dot{\rho}_{m}+\dot{\rho}_{\Lambda}+3 H\left(\rho_{m}+\rho_{\Lambda}+p_{\Lambda}\right)=0
$$

It is assumed that the dark matter component is interacting with the dark energy through an interacting term $Q$, the continuity equation of matter and dark energy can be obtained as,

$$
\dot{\rho}_{m}+\left(\frac{\dot{V}}{V}\right) \rho_{m}=Q
$$




$$
\dot{\rho}_{\Lambda}+\left(\frac{\dot{V}}{V}\right)\left(1+\omega_{\Lambda}\right) \rho_{\Lambda}=-Q
$$

where $\omega_{\Lambda}=\frac{p_{\Lambda}}{\rho_{\Lambda}}$ is the equation of state parameter for the holographic dark energy and $Q>0$ measures the strength of interaction. A vanishing $Q$ implies that the dark matter and dark energy are separately conserved. In view of continuity equations, the interaction between dark energy and dark matter must be a function of the energy density multiplied by a quantity, with units of inverse of time, which can be chosen as the Hubble parameter H. It's a freedom to choose the form of energy density which can be any combination of dark energy and dark matter. Thus interaction between dark energy and dark matter could be expressed phenomenologically in the form as (Guo et al. [43] [44], Amendola et al. [63]),

$$
Q=3 b^{2} H \rho_{m}=b^{2} \frac{\dot{V}}{V} \rho_{m}
$$

where $b^{2}$ is the coupling constant.

Cai and Wang [64] have taken the same relation for interacting dark matter and phantom dark energy in order to avoid the coincidence problem.

Using Equations (2.15) and (2.17), we get the energy density of dark matter as,

$$
\rho_{m}=\rho_{0} V^{\left(b^{2}-1\right)}
$$

where $\rho_{0}>0$ is a real constant of integration.

Using Equations (2.17) and (2.18), we get the interacting term $Q$ as,

$$
Q=3 \rho_{0} b^{2} H V^{\left(b^{2}-1\right)}
$$

\section{Cosmological Solution for Variable Deceleration Parameter}

We consider the deceleration parameter to be a variable

$$
q=-\frac{a \ddot{a}}{\dot{a}^{2}}=-\frac{\ddot{a}}{a H^{2}}=b(t)
$$

and following Pradhan et al. [65] and Chawla et al. [1], we assume the law of variation of scale factor as increasing function of time

$$
a(t)=[\sinh (\alpha t)]^{\frac{1}{n}}
$$

Using (3.2) in Equations (2.12a)-(2.12c), we obtain the expressions for scale factors as,

$$
\begin{gathered}
a_{1}(t)=V^{1 / 3}=a=(\sinh \alpha t)^{1 / n} \\
a_{2}(t)=D(\sinh \alpha t)^{1 / n} \exp \left(X \int \frac{\mathrm{d} t}{(\sinh \alpha t)^{1 / n}}\right)
\end{gathered}
$$




$$
a_{3}(t)=D^{-1}(\sinh \alpha t)^{1 / n} \exp \left(-X \int \frac{\mathrm{d} x}{(\sinh \alpha t)^{1 / n}}\right),
$$

where $X$ and $D$ are constants of integration.

Using Equations (2.10) and (3.2) in Equations (2.17) and (2.18), we obtained,

$$
\begin{gathered}
Q=\frac{3}{n} \rho_{0} b^{2} \alpha \operatorname{coth} \alpha t(\sinh (\alpha t))^{\frac{3}{n}\left(b^{2}-1\right)}, \\
\rho_{m}=\rho_{0}(\sinh (\alpha t))^{\frac{3}{n}\left(b^{2}-1\right)} .
\end{gathered}
$$

Using Equations (3.2)-(3.5) and (3.7) in Equation (2.4) we obtained the density of holographic dark energy as,

$$
\rho_{\Lambda}=\frac{3 \alpha^{2}}{n^{2}} \operatorname{coth}^{2}(\alpha t)-\left(X^{2}+\beta^{2}\right)\left[\frac{1}{\sinh (\alpha t)^{1 / n}}\right]^{2}-\rho_{0}(\sinh (\alpha t))^{\frac{3}{n}\left(b^{2}-1\right)}
$$

Using Equation (3.3)-(3.5) in Equation (2.7), we obtained the pressure as,

$$
p_{\Lambda}=\frac{\alpha^{2}}{n^{2}}\left[(2 n-3) \operatorname{coth}^{2}(\alpha t)-2 n\right]-\frac{2 \alpha X}{n} \frac{\operatorname{coth}(\alpha t)}{(\sinh (\alpha t))^{\frac{1}{n}}}+\frac{\beta^{2}-X^{2}}{(\sinh (\alpha t))^{\frac{2}{n}}} \text {. }
$$

The EOS parameter $\omega_{\Lambda}$ of holographic dark energy is given by,

$$
\omega_{\Lambda}=\frac{\alpha^{2}\left[(2 n-3) \operatorname{coth}^{2}(\alpha t)-2 n\right](\sinh (\alpha t))^{\frac{2}{n}}-2 n \alpha X \operatorname{coth}(\alpha t)(\sinh (\alpha t))^{\frac{1}{n}}+n^{2}\left(\beta^{2}-X^{2}\right)}{3 \alpha^{2} \operatorname{coth}^{2}(\alpha t)(\sinh (\alpha t))^{\frac{2}{n}}-n^{2} \rho_{0}(\sinh (\alpha t))^{\frac{1}{n}\left(3 b^{2}-1\right)}-n^{2}\left(\beta^{2}+X^{2}\right)}
$$

The physical parameters such as spatial volume $V$, Hubble parameter $H$, expansion scalar $\theta$ and the time varying deceleration parameter $q$ are obtained as,

$$
\begin{gathered}
V=a^{3}=(\sinh (\alpha t))^{3 / n} \\
H=\frac{\alpha}{n} \operatorname{coth}(\alpha t) \\
\theta=3 H=\frac{3 \alpha}{n} \operatorname{coth}(\alpha t) \\
q=n\left(1-\tanh ^{2}(\alpha t)\right)-1 .
\end{gathered}
$$

The shear scalar $\sigma$ and mean isotropy parameter $\Delta$ are given by,

$$
\begin{gathered}
\sigma^{2}=\frac{1}{6} \theta^{2}=\frac{3 \alpha^{2}}{2 n^{2}} \operatorname{coth}^{2}(\alpha t), \\
\Delta=\frac{1}{3} \sum_{i=1}^{3}\left(\frac{H_{i}-H}{H}\right)^{2}=\frac{2 n^{2} X^{2}(\sinh (\alpha t))^{-2 / n}}{3 \alpha^{2} \operatorname{coth}^{2}(\alpha t)} .
\end{gathered}
$$

\section{Conclusions}

In this paper, we have presented spatially homogeneous and anisotropic Bianchi Type-V universe field with interacting dark matter and holographic dark energy. 
With the consideration of variable deceleration parameter, we obtained the solutions of Einstein's field equations.

It is found that the universe approaches to isotropy for large cosmic time as shown by different observational data and dark energy is responsible for expansion of universe. The concluding remarks of the model are as follows.

1) The sign of $q$ represents that the universe is decelerating or accelerating i.e. a positive sign of $q$ represents accelerating universe and negative sign of $q$ represents decelerating universe.

In our model $q>0$ for $t \rightarrow 0$ and $q \leq-1$ for $t \rightarrow \infty$ i.e. the model represents the decelerating to accelerating phase and the values of deceleration parameter lie in the phase $-1 \geq q>0$.

2) From the Equation (3.11), we can say that the spatial volume $V$ is finite at $t=$ 0 and expands as $t$ increases and becomes infinite for $t=\infty$.

3) From Equation (3.16), we can conclude that for the large cosmic time (i.e. $t \rightarrow \infty)$, the anisotropy parameter $\Delta \rightarrow 0$. Therefore, for the large cosmic time, the anisotropy of the universe damped out and the universe approaches to an isotropy universe.

4) From the Equation (3.12), it is observed that the directional Hubble parameter diverges for $t=0$ and converges for $t=\infty$.

5) We observe that $p_{\Lambda}$ (i.e. pressure of dark energy) tends to a negative value for large cosmic time which shows that the universe is accelerating (SNeIa).

6) From the Equation (3.10), the EOS parameter $\omega_{\Lambda}<-1$ for large cosmic time. In this case, the holographic dark energy looks like phantom energy, (Abazajian et al. [15], Ade et al. [66], Riess et al. [4] [67], Zimdahl [51]). Our result is consistent with SNeIa.

7) For $\beta=0$ in Equation (2.1), the investigated model approaches to Mete, et al. [68].

\section{Conflicts of Interest}

The authors declare no conflicts of interest regarding the publication of this paper.

\section{References}

[1] Chawla, C., Mishra, R.K. and Pradhan, A. (2012) Anisotropic Bianchi-I Cosmological Models in String Cosmology with Variable Deceleration Parameter. Romanian Journal of Physics, 58, 1000. arXiv:1203.4014 [physics.gen-ph]

[2] Perlmutter, S.J., et al. (1998) Discovery of a Supernova Explosion at Half the Age of the Universe. Nature, 391, 51-54. https://doi.org/10.1038/34124

[3] Perlmutter, S.J., et al. (1999) Measurements of $\Omega$ and $\Lambda$ from 42 High-Redshift Supernovae. The Astrophysical Journal, 517, 565-586. https://doi.org/10.1086/307221

[4] Riess, A.G., et al. (1998) Observational Evidence from Supernovae for an Accelerating Universe and a Cosmological Constant. The Astronomical Journal, 116, 1009-1038. https://doi.org/10.1086/300499 
[5] Riess, A.G., et al. (2004) Type Ia Supernova Discoveries at $z>1$ from the Hubble Space Telescope: Evidence for Past Deceleration and Constraints on Dark Energy Evolution. The Astrophysical Journal, 607, 665-687. https://doi.org/10.1086/383612

[6] Astier, P., et al. (2006) The Supernova Legacy Survey: Measurement of $\Omega_{\mathrm{M}}, \Omega_{\Lambda}$ and from First Year Data Set. Astronomy \& Astrophysics, 447, 31-48. https://doi.org/10.1051/0004-6361:20054185

[7] Spergel, D.N., et al. (2006) Three-Year Wilkinson Microwave Anisotropy Probe (WMAP) Observations: Implications for Cosmology. The Astrophysical Journal, 170, 377. https://doi.org/10.1086/513700

[8] Davis, T.M., et al. (2007) Scrutinizing Exotic Cosmological Models Using Essence Supernova Data Combined With Other Cosmological Probes. The Astrophysical Journal, 666, 716-725. https://doi.org/10.1086/519988

[9] Bennett, C.L., Halpern, M., Hinshaw, G., Jarosik, N., Kogut, A., et al. (2003) First Year Wilkinson Microwave Anisotropy Probe (WMAP) Observations: Preliminary Maps and Basic Results. The Astrophysical Journal, 148, 1-43.

https://doi.org/10.1086/377253

[10] Spergel, D.N., Verde, L., Peiris, H.V., Komatsu, E., Nolta, M.R., et al. (2003) First Year Wilkinson Microwave Anisotropy Probe (WMAP) Observations Determination of Cosmological Parameters. The Astrophysical Journal, 148, 175-194. https://doi.org/10.1086/377226

[11] Verde, L., et al. (2002) The 2dF Galaxy Redshift Survey: The Bias of Galaxies and the Density of the Universe. Monthly Notices of the Royal Astronomical Society, 335, 432-440. https://doi.org/10.1046/j.1365-8711.2002.05620.x

[12] Hawkins, E., et al. (2003) The 2dF Galaxy Redshift Survey: Correlation Functions, Peculiar Velocities and the Matter Density of the Universe. Monthly Notices of the Royal Astronomical Society, 346, 78-96. https://doi.org/10.1046/j.1365-2966.2003.07063.x

[13] Abazajian, K., et al. (2003) The First Data Release of The Sloan Digital Sky Survey. The Astronomical Journal, 126, 2081-2086. https://doi.org/10.1086/378165

[14] Abazajian, K., et al. (2004) The Second Data Release of the Sloan Digital Sky Survey. The Astronomical Journal, 128, 502-512.

[15] Abazajian, K., et al. (2004) The Third Data Release of The Sloan Digital Sky Survey. The Astronomical Journal, 129, 1755-1759.

[16] Tegmark, M., et al. (2004) Cosmological Parameters from SDSS and WMAP. Physical Review D, 69, Article ID: 103501. https://doi.org/10.1103/PhysRevD.69.103501

[17] Weinberg, S. (1989) The Cosmological Constant Problem. Review of Modern Physics, 61, 1. https://doi.org/10.1103/RevModPhys.61.1

[18] Carroll, S.M. (2001) The Cosmological Constant. Living Reviews in Relativity, 4, 1. https://doi.org/10.12942/lrr-2001-1

[19] Peebles, P.J.E. and Ratra, B. (2003) The Cosmological Constant and Dark Energy. Reviews of Modern Physics, 75, 559. https://doi.org/10.1103/RevModPhys.75.559

[20] Padmanabhan, T. (2003) Cosmological Constant: The Weight of the Vacuum. Physics Reports, 380, 235-320. https://doi.org/10.1016/S0370-1573(03)00120-0

[21] Koivisto, T. and Mota, D.F. (2008) Anisotropic Dark Energy: Dynamics of the Background and Perturbations. Journal of Cosmology and Astroparticle Physics, 2008, 18. https://doi.org/10.1088/1475-7516/2008/06/018

[22] Cohen, A.G., Kaplan, D.B. and Nelson, A.E. (1999) Effective Field Theory, Black Holes, and the Cosmological Constant. Physics Review Letters, 82, 4971-4974. 
https://doi.org/10.1103/PhysRevLett.82.4971

[23] Horava, P. and Minic, D. (2000) Probable Values of the Cosmological Constant in a Holographic Theory. Physical Review Letters, 85, 1610.

https://doi.org/10.1103/PhysRevLett.85.1610

[24] Thomas, S. (2002) Holography Stabilizes the Vacuum Energy. Physical Review Letters, 89, Article ID: 081301. https://doi.org/10.1103/PhysRevLett.89.081301

[25] Li, M. (2004) A Model of Holographic Dark Energy. Physics Letters B, 603, 1-5. https://doi.org/10.1016/j.physletb.2004.10.014

[26] Hooft, G. (1995) Dimensional Reduction in Quantum Gravity. General Relativity and Quantum Cosmology (gr-qc), 9310026.

[27] Coles, P. and Eills, G.F.R. (1994) The Case for an Open Universe. Nature, 370, 609-615. https://doi.org/10.1038/370609a0

[28] Zhang, X. and Wu, F.Q. (2005) Constraints on Holographic Dark Energy from Type Ia Supernova Observations. Physical Review D, 72, Article ID: 043524. https://doi.org/10.1103/PhysRevD.72.043524

[29] Enqvist, K., Hannestad, S. and Sloth, M.S. (2005) Searching for a holographic connection between Dark Energy and the Low/CMB Multipoles. Journal of Cosmology and Astroparticle Physics, 2005, 4. https://doi.org/10.1088/1475-7516/2005/02/004

[30] Shen, J., Wang, B., Abdalla, E. and Su, R.-K. (2005) Constraints on the Dark Energy from the Holographic Connection to the Small $1 \mathrm{CMB}$ Suppression. Physics Letters $B, 609,200-205$. https://doi.org/10.1016/j.physletb.2005.01.051

[31] Chang, Z., Wu, F.-Q. and Zhang, X. (2006) Constraints on Holographic Dark Energy from X-Ray Gas Mass Fraction of Galaxy Clusters. Physics Letters B, 633, 14-18. https://doi.org/10.1016/j.physletb.2005.10.095

[32] Carvalho, F.C. and Saa, A. (2004) Nonminimal Coupling, Exponential Potentials and the $\omega<-1$ Regime of Dark Energy. Physical Review D, 70, Article ID: 087302. https://doi.org/10.1103/PhysRevD.70.087302

[33] Huang, Q.-G. and Li, M. (2004) The Holographic Dark Energy in a Non-Flat Universe. Journal of Cosmology and Astroparticle Physics, 2004, 13. https://doi.org/10.1088/1475-7516/2004/08/013

[34] Gong, Y.-G. (2004) Extended Holographic Dark Energy. Physical Review D, 70, Article ID: 064029. https://doi.org/10.1103/PhysRevD.70.064029

[35] Gong, Y.-G. and Zhang, Y.-Z. (2005) Holography and Holographic Dark Energy Model. Classical and Quantum Gravity, 22, 4895-4902. https://doi.org/10.1088/0264-9381/22/22/014

[36] Pavon, D. and Zimdahl, W. (2005) Holographic Dark Energy and Cosmic Coincidence. Physics Letters B, 628, 206-210. https://doi.org/10.1016/j.physletb.2005.08.134

[37] Wang, B., Gong, Y.G. and Abdalla, E. (2005) Transition of the Dark Energy Equation of State in an Interacting Holographic Dark Energy Model. Physics Letters B, 624, 141-146. https://doi.org/10.1016/j.physletb.2005.08.008

[38] Perivolaropoulos, L. (2005) Crossing the Phantom Divide Barrier with Scalar Tensor Theories. Journal of Cosmology and Astroparticle Physics, 2005, 1. https://doi.org/10.1088/1475-7516/2005/10/001

[39] Nojiri, S. and Odintsov, S.D. (2006) Unifying Phantom Inflation with Late-Time Acceleration: Scalar Phantom-Non-Phantom Transition Model and Generalized Holographic Dark Energy. General Relativity and Gravitation, 38, 1285-1304. https://doi.org/10.1007/s10714-006-0301-6 
[40] Guberina, B., Horvat, R. and Nikolic, H. (2005) Generalized Holographic Dark Energy and the IR Cutoff Problem. Physics Review D, 72, Article ID: 125011. https://doi.org/10.1103/PhysRevD.72.125011

[41] Guberina, B., Horvat, R. and Nikolić, H. (2006) Dynamical Dark Energy with a Constant Vacuum Energy Density. Physics Letters B, 636, 80-85. https://doi.org/10.1016/j.physletb.2006.03.041

[42] Guo, Z. K., Ohta, N. and Zhang, Y.Z. (2005) Parameterization of Quintessence and Its Potential. Physical Review D, 72, Article ID: 023504. https://doi.org/10.1103/PhysRevD.72.023504

[43] Guo, Z.K., Ohta, N. and Tsujikawa, S. (2007) Probing the Coupling between Dark Components of the Universe. Physics Review D, 76, Article ID: 023508. https://doi.org/10.1103/PhysRevD.76.023508

[44] Guo, Z.K., Ohta, N. and Zhang, Y.Z. (2007) Parametrizations of the Dark Energy Density and Scalar Potentials. Modern Physics Letters A, 22, 883-890. https://doi.org/10.1142/S0217732307022839

[45] Hu, B. and Ling, Y. (2006) Interacting Dark Energy, Holographic Principle, and Coincidence Problem. Physical Review D, 73, Article ID: 123510. https://doi.org/10.1103/PhysRevD.73.123510

[46] Li, H., Guo, Z.K. and Zhang, Y.Z. (2006) A Tracker Solution for a Holographic Dark Energy Model. International Journal of Modern Physics D, 15, 869-877. https://doi.org/10.1142/S0218271806008577

[47] Setare, M.R. (2006) Interacting Holographic Dark Energy Model in Non-Flat Universe. Physics Letters B, 642, 1-4. https://doi.org/10.1016/j.physletb.2006.09.027

[48] Setare, M.R. (2007) The Holographic Dark Energy in Non-Flat Brans-Dicke Cosmology. Physics Letters B, 644, 99-103. https://doi.org/10.1016/j.physletb.2006.11.033

[49] Sadjadi, H.M. (2007) The Particle versus the Future Event Horizon in an Interacting Holographic Dark Energy Model. Journal of Cosmology and Astroparticle Physics, 2007, 26. https://doi.org/10.1088/1475-7516/2007/02/026

[50] Banerjee, N. and Pavon, D. (2007) Holographic Dark Energy in Brans-Dicke Theory. Physics Letters B, 647, 477-481. https://doi.org/10.1016/j.physletb.2007.02.035

[51] Zimdahl, W. (2008) Dark Energy: A Unifying View. International Journal of Modern Physics D, 17, 651-658. https://doi.org/10.1142/S0218271808012395

[52] Sarkar, S. (2014) Holographic Dark Energy Model with Linearly Varying Deceleration Parameter and Generalised Chaplygin Gas Dark Energy Model in Bianchi Type-I Universe. Astrophysics and Space Science, 349, 985-993. https://doi.org/10.1007/s10509-013-1684-y

[53] Sarkar, S. (2014) Interacting Holographic Dark Energy with Variable Deceleration Parameter and Accreting Black Holes in Bianchi Type-V Universe. Astrophysics and Space Science, 352, 245-253. https://doi.org/10.1007/s10509-014-1876-0

[54] Sarkar, S. (2014) Interacting Holographic Dark Energy with Variable Deceleration Parameter and Tachyon Scalar Field Dark Energy Model in LRS Bianchi Type-II Universe. Astrophysics and Space Science, 350, 821-829. https://doi.org/10.1007/s10509-014-1786-1

[55] Adhav, K.S. (2011) Statefinder Diagnostic for Variable Modified Chaplygin Gas in Bianchi Type-V Universe. Astrophysics and Space Science, 335, 611-617. https://doi.org/10.1007/s10509-011-0773-z 
[56] Adhav, K.S. (2011) Statefinder Diagnostic for Modified Chaplygin Gas in Bianchi Type-V Universe. The European Physical Journal Plus, 126, 52. https://doi.org/10.1140/epjp/i2011-11052-6

[57] Pradhan, A., Amirhashchi, H. and Saha, B. (2011) Bianchi Type-I Anisotropic Dark Energy Model with Constant Deceleration Parameter. International Journal of Theoretical Physics, 50, 2923-2938. https://doi.org/10.1007/s10773-011-0793-z

[58] Kumar, S. and Yadav, A.K. (2011) Some Bianchi Type-V Models of Accelerating Universe with Dark Energy. Modern Physics Letters A, 26, 647-659. https://doi.org/10.1142/S0217732311035018

[59] Pradhan, A. and Amirhashchi, H. (2011) Accelerating Dark Energy Models in Bianchi Type-V Spacetime. Modern Physics Letters A, 26, 2266-2275. https://doi.org/10.1142/S0217732311036620

[60] Adhav, K.S., Tayade, G.B. and Bansod, A.S. (2014) Interacting Dark Matter and Holographic Dark Energy in an Anisotropic Universe. Astrophysics and Space Science, 353, 249-257. https://doi.org/10.1007/s10509-014-2015-7

[61] Som, S. and Sil, A. (2014) Interacting Holographic Dark Energy Models: A General Approach. Astrophysics and Space Science, 352, 867-875. https://doi.org/10.1007/s10509-014-1926-7

[62] Granda, L.N. and Oliverios, A. (2008) Infrared Cut-Off Proposal for the Holographic Density. Physics Letters B, 669, 275-277. https://doi.org/10.1016/j.physletb.2008.10.017

[63] Amendola, L., Campos, G.C. and Rosenfeld, R. (2007) Consequences of Dark Matter-Dark Energy Interaction on Cosmological Parameters Derived from Type Ia Supernova Data. Physical Review D, 75, Article ID: 083506. https://doi.org/10.1103/PhysRevD.75.083506

[64] Cai, R.-G. and Wang, A. (2005) Cosmology with Interaction between Phantom Dark Energy and Dark Matter and the Coincidence Problem. Journal of Cosmology and Astroparticle Physics, 2005, 2. https://doi.org/10.1088/1475-7516/2005/03/002

[65] Pradhan, A., Jaiswal, R., Jotania, K. and Khare, R.K. (2012) Dark Energy Models with Anisotropic Fluid in Bianchi Type- $V I_{0}$ Space-Time with Time Dependent Deceleration Parameter. Astrophysics and Space Science, 337, 401-413. https://doi.org/10.1007/s10509-011-0835-2

[66] Ade, P.A.R., et al. (2014) Plank 2013 Results XVI Cosmological Parameters. Astronomy and Astrophysics, 571, 1-66.

[67] Riess, A.G., et al. (2007) New Hubble Space Telescope Discoveries of Type Ia Supernovae at $\mathrm{z} \geq 1$ : Narrowing Constraints on the Early Behavior of Dark Energy. Astrophysical Journal, 659, 98-121. https://doi.org/10.1086/510378

[68] Mete, V.G., et al. (2018) Interacting Dark Fluids In Bianchi Type-I Universe With Variable Deceleration Parameter. International Journal of Current Advanced Research, 7, 13609-13613. 